\title{
Investigating the Relationship Between the Hot Gas and the Dark Matter Components of Galaxy Clusters.
}

\author{
Leila C. Powell ${ }^{1}$, Scott T. Kay ${ }^{2}$, Arif Babul ${ }^{3}$ \\ and Andisheh Mahdavi ${ }^{3}$ \\ ${ }^{1}$ Oxford Astrophysics, Denys Wilkinson Building, Keble Road, Oxford, OX1 3RH, UK \\ email: lcp@astro.ox.ac.uk \\ ${ }^{2}$ Jodrell Bank Observatory, University of Manchester, Macclesfield, Cheshire, SK11 9DL, UK \\ ${ }^{3}$ University of Victoria, Department of Physics and Astronomy, Victoria, V8P 5C2, Canada
}

\begin{abstract}
Various differences in galaxy cluster properties derived from X-ray and weak lensing observations have been highlighted in the literature. One such difference is the observation of mass concentrations in lensing maps which have no X-ray counterparts (e.g. Jee, White, Ford et al. 2005). We investigate this issue by identifying substructures in maps of projected total mass (analogous to weak lensing mass reconstructions) and maps of projected X-ray surface brightness for three simulated clusters. We then compare the $2 \mathrm{D}$ mass substructures with both $3 \mathrm{D}$ subhalo data and the 2D X-ray substructures. Here we present preliminary results from the first comparison, where we have assessed the impact of projecting the data on subhalo identification.
\end{abstract}

Keywords. X-rays: galaxies: clusters, gravitational lensing, methods: numerical

\section{Introduction}

Six clusters from a $\Lambda \mathrm{CDM}$ cosmological simulation (performed by the Virgo Consortium†), are resimulated, using GADGET-2 (Springel 2005), with $10^{5}$ particles (low resolution) and a non-radiative gas physics model. In order that we can later assess the impact of dynamical state, three are selected for their varied mass histories and are also resimulated with $\sim 1 \times 10^{6}$ particles (high resolution). We examine the clusters within $r_{500}$ as this is an approximate upper limit on the extent of X-ray observations.

\section{Identifying substructures in $2 \mathrm{D}$ and $3 \mathrm{D}$}

The maps are smoothed with a Gaussian filter of $\mathrm{FWHM}=60 \mathrm{kpc} \mathrm{h}^{-1}$ to remove structures smaller than the simulation's force resolution and reduce random noise. The substructures in the maps are then enhanced using an unsharpmasking technique to remove the cluster background. The enhanced map is searched for substructures with greater than $3 \sigma$ significance, that are then characterised by ellipses. This technique is effective for relaxed and disturbed clusters as it does not rely on circular symmetry.

Positions and masses of self-bound 3D subhaloes are obtained using a version of SUBFIND (Springel, White, Tormen et al. 2001) and these are then cross-referenced with the catalogues of $2 \mathrm{D}$ mass substructures. A subhalo is considered a match to a source in the map if its centre lies within the ellipse that characterises the source (see figure 1 for

\section{$\dagger$ http://www.virgo.dur.ac.uk}


matching success). If more than one subhalo satisfies this criteria, the source in the map is flagged as a multiple match and is assigned the combined mass of all the subhaloes.

\section{The effect of projection on substructure identification}

We find a clear correlation between the area of the substructure in the enhanced projected mass map and the subhalo mass(es) attributed to it after matching with the $3 \mathrm{D}$ data (figure 2). We apply a mass limit to the multiple matching technique, such that further subhaloes can only be matched to a source if they have a given mass ratio with the most massive matched subhalo. A significant number of multiple matches are found only if the limiting mass ratio is reduced to 100:1. This indicates that genuine projection effects are not an issue in our sample.

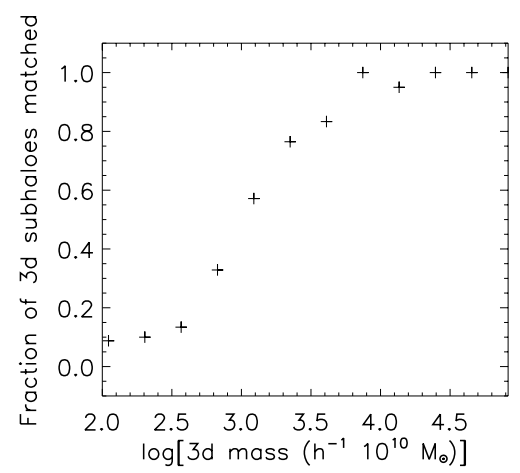

Figure 1. Dependence of matching success of $3 \mathrm{D}$ subhaloes to $2 \mathrm{D}$ substructures in the projected mass map on subhalo mass (all clusters combined).

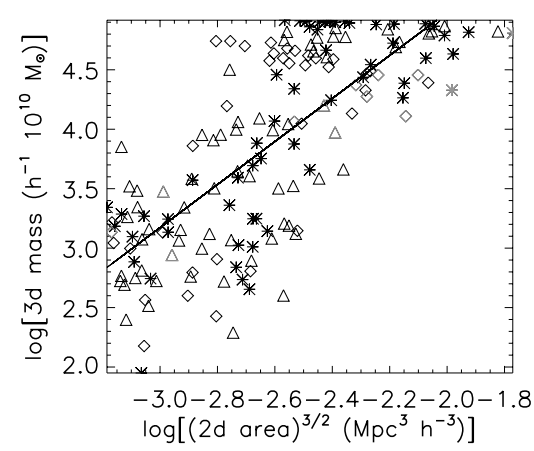

Figure 2. Correlation between area of $2 \mathrm{D}$ substructure in map and associated 3D subhalo mass for cluster 1 (asterix), cluster 2 (diamonds) and cluster 3 (triangles). $2 \mathrm{~d}$ substructures which are a multiple match (with 100:1 mass limit) are shown in grey.

\section{Future work}

We will investigate the impact of confusion, by varying the FWHM of the Gaussian filter and examining the effect on the probability of multiple matches. By comparing the $2 \mathrm{D}$ mass substructures to the 2D X-ray substructures, we can establish how the two components differ and how this depends on 3D subhalo mass, dynamical state, position in the cluster and redshift. This comparison will be extended to the high resolution simulations and to simulations including feedback and cooling (Powell et al., in preparation).

\section{Acknowledgements}

LCP is supported by an STFC studentship.

\section{References}

Jee, M. J., White, R. L., Ford, H. C., Blakeslee, J. P., Illingworth, G. D., Coe, D. A. \& Tran, K.-V. H. 2005, ApJ 634, 813-832

Springel, V. 2005, MNRAS 364, 1105-1134

Springel V., White, S. D. M., Tormen, G. \& Kauffmann, G. 2001, MNRAS 328, 726-750 